\title{
Primary Histoplasmosis of Larynx: A Case Series and Review of Literature
}

\author{
Nishi Sonkhya ${ }^{1 *}$, Raghav Mehta ${ }^{1}$, Divij Sonkhya ${ }^{2}$, Shubha Gupta ${ }^{3}$, Mansi Faujdar ${ }^{3}$ \\ ${ }^{1}$ Department of Ear, Nose and Throat, S.M.S. Medical College and Hospital, Jaipur, India \\ ${ }^{2}$ Department of Ear, Nose and Throat, N.K.P. Salve Institute \& Lata Mangeshker Hospital, Jaipur, India \\ ${ }^{3}$ S.D.M. Hospital, Jaipur, India \\ Email: *drsonkhya@yahoo.co.in, drraghavmehta@rediffmail.com,divijsonkhya@yahoo.co.in, \\ drshubhagupta01@gmail.com,mansifaujdar@yahoo.co.in
}

Received February 5, 2013; revised March 10, 2013; accepted March 20, 2013

\begin{abstract}
Objective: Aim of the study was to analyze 10 managed cases of Primary Histoplasmosis of Larynx, over a period of 10 years. Design: Retrospective, non-randomized, interventional case series. Patients and Method: This study was conducted in the Department of Otorhinolaryngology S.M.S. Medical College from 2002 to 2011. The study consisted of evaluation of self-reported and referred patients who presented to ENT OPD and emergency. In all the cases a detailed history and clinical examination was done. All the patients underwent fiber optic laryngoscopy and subsequent direct laryngoscopy and biopsy. CT Scans were obtained for few cases. The study was a retrospective analysis of 10 treated cases. Main Outcome and Measures: Success of the treatment was defined as resolution of symptoms, differentiation from malignancy, no recurrence and regular follow up. Result: A total of 10 cases were managed between 2002 and 2011 and were reviewed. The main presentation was hoarseness of voice, with lesion simulating malignancy. Most common site of involvement was false cord and aryepiglottic fold (6 cases). Primary laryngeal Histoplasmosis was essentially a disease of adult males in all 10 cases. Treatment was exclusively medical with use of Itraconazole as drug of choice in 7 cases. Conclusion: Isolated laryngeal Histoplasmosis is a rare entity. Because of its simulation with malignancy it needs to be included in the differential diagnosis of neoplasm both benign and malignant. Our study clearly limits this kind of disease to adult males most commonly involving false cord and aryepiglottic fold in an endophytic pattern. Though it looks like cancer; biopsy confirms the diagnosis. The treatment is mainly medical with Itraconazole being the drug of choice.
\end{abstract}

Keywords: Laryngeal Histoplasmosis; Histoplasma Capsulatum; Laryngeal Malignancy

\section{Introduction}

Histoplasmosis (also known as "Cave disease," "Darling’s disease," "Ohio valley disease," "Reticuloendotheliosis," "Spelunker's Lung” and Caver's disease) [1] is a disease caused by the fungus Histoplasma capsulatum. Histoplasmosis occurs throughout the world but is more common within endemic areas of North America, particularly in fertile river valleys. It can produce a spectrum of illness, from subclinical infection of lung to progressive disseminated diseases [2]. The vast majority of infected people are asymptomatic (have no apparent ill effects), or they experience symptoms so mild that they do not seek medical attention. If symptoms do occur, they will usually start within 3 to 17 days after exposure, with an average of 10 days. Chronic histoplasmosis cases can resemble tuberculosis, [3] disseminated histoplasmosis affects multiple organ systems and is fatal unless

\footnotetext{
${ }^{*}$ Corresponding author.
}

treated [4].

Histoplasmosis can appear as a mild, flu-like respiretory illness and has a combination of symptoms, including malaise (a general ill feeling), fever, chest pain, dry or nonproductive cough, headache, loss of appetite, shortness of breath, joint and muscle pains, chills, and hoarseness. A chest X-ray can reveal distinct markings on an infected person's lungs.

Though primary infection of larynx is a rarity and generally associated with generalized disseminated disease, we have seen and collected 10 cases over a period of 10 years, which presented to us primarily affecting the larynx. On initial assessment the patients were diagnosed as carcinoma larynx but on progressive histopathological examination diagnosis of histoplasmosis was confirmed.

Laryngeal involvement occurs in the mucocutaneous form of the chronic disease. Common features are: pain to swallow, hoarseness, gingival ulceration and dysphagia. There can be weight loss, malaise and fatigue. As per 
the data histoplasmosis of head and neck is more common in adults and exclusively in males.

\section{Method}

This study was conducted in the Department of Otorhinolaryngology S.M.S. Medical College from 2002 to 2011. The study consisted of evaluation of self-reported and referred patients who presented to ENT OPD and emergency. In all the cases a detailed history regarding the complaints was taken which included elaboration of all the complaints with regards to onset, duration and progress. A through clinical examination was done which included a comprehensive Ear, Nose and Throat examination. Special care was done to examine the oral cavity, oropharynx, pharynx, larynx and neck as these were the main complaint areas. Indirect laryngoscopy was done in all cases in first sitting and then subsequently all the patients underwent fiber optic laryngoscopy. Then patient was prepared for General anesthesia by getting all the relevant blood examination, which also helped in ruling out any acute infection or other blood related maladies. The OT was prepped and a direct laryngoscopy was done to further confirm the gross feature of the lesion and further taking biopsy to confirm the diagnosis histopathologically. Contrast enhanced CT Scans were obtained for few cases and radiologist was specifically asked to give thinner cuts at the lesion site. The study was a retrospective analysis of 10 treated cases.

\section{Results and Analysis}

Out of 10 cases, 8 cases were in age group of 40 to 60 years. Youngest patient was 32 years old and eldest one was 60 years of age. Disease was exclusively seen in males. The most common presenting symptom was gradually progressing hoarseness of voice (10 cases), and difficulty in swallowing (3 cases). Laryngeal examination revealed endophytic growth in 6 cases, exophytic growth in 2 cases and ulcerative lesion in 2 cases. False cord and aryepiglottic fold was the common site of involvement (6 cases). Epiglottis involvement was seen in 3 cases and only 1 case was with postcricoid lesion.

Histopathological diagnosis was mandatory in all the cases. A sufficient quantity of tissue was taken and sent to the pathologist in all the sterile conditions with 6 hours of the biopsy The pathologist did two types of staining to confirm the diagnosis. Both Hematoxyline \& Eosin and Silver methenamine stains (MSN) showed Histiocytes (macrophages) with Histoplasma, granuloma with caseation and pseudoepiteliomatous hyperplasia.

In the past Amphotericin B was the drug of choice, which was used in 3 cases. We have used Itraconazole $200 \mathrm{mg}$, twice a day, (by mouth) in 7 cases. All cases were successfully treated on 6 months follow-up. Reso- lution of clinical symptoms and clearance of the positive culture was obtained in these cases. There were no signs of pulmonary or Systemic involvement.

\section{Discussion}

Histoplasmosis is a dimorphic fungal infection first described and named by Darling in 1906. Initally he thought causative agent was a protozoan, later it proved to be a fungus. Though fungal nature was confirmed in early stages it was treated as a deficiency disease [5]. The pathogenesis of histoplasmosis is incompletely understood. Fungus expresses heat shock protein 60 (hsp 60) on its cell surface that binds to alpha 2 integrins on the surface of macrophages. Histoplasma induces macrophages to secrete tumor necrosis factor (TNF), which stimulates and recruits other macrophages to kill the Histoplasma [6]. It has two distinct growth forms-yeast and mycelia forms. Yeast forms occur in humans and soil. On microscopy branched hyphae are seen. Hyphae have tubercular projections which contains conidias. These conidias are found in soil and are inhaled by humans to give rise to pulmonary infection. Rapid transient hematogenous and systemic spread then occurs. But in many cases it may remain restricted to lungs forming residual calcification.

Clinically it can be classified into 4 types according to Furcolow [7].

1) Acute Pulmonary Histoplasmosis;

2) Chronic pulmonary histoplasmosis-progressive cavitary;

3) Acute Disseminated Histoplasmosis-benign progressive;

4) Chronic Disseminated Histoplasmosis-Mucocutaneous, Meningitis, Pericarditis, Adrenal insufficiency and Bone marrow involvement.

The source of infection is usually the soil with high nitrogen content which is result of contamination by bat and bird guano. Thus found in areas of bird roosting, caves and chicken houses.

Histoplasma capsulatum infection involving the larynx is a rare manifestation, especially in immunocompetent individuals and a high index of suspicion is needed to establish the diagnosis correctly [8]. Hoarseness, dysphagia and pain on swallowing are symptoms common to many diseases of larynx and laryngopharynx, including tuberculosis, histoplasmosis, papillomatous lesion, carcinoma and other granulomatous lesion. It is therefore important to consider fungus in the differential diagnosis [9].

In our series all the 10 cases presented with Hoarseness of voice as main symptom. Difficulty in swallowing was also one of the features in 2 cases. Subramaniam (2005) [10], Pochini Sobrinho (2007) [11] and Katoch 
(2009) [12] reported that main presenting symptom in patients of primary laryngeal histoplasmosis is hoarseness of voice followed by pain to swallow and dysphagia.

As per the study we could see that primary laryngeal Hiatoplasmosis was a disease of adult males with around $80 \%$ cases between age group of 40 - 60 . In all the previous reported cases males were exclusively involved. Subramaniam [10] and Donegan [13] reported one case each in 52 and 69 years old males. Katoch [12] reported a case in 20 year old male.

The most common site to be involved in our study was false cord and aryepiglottic fold (Figure 1), followed by laryngeal surface of epiglottis. Involvement of vocal cords [10], laryngeal surface of epiglottis [11], post cricoid region [12], vallecula, pharyngoepiglottic fold [14] and supraglottic region [15] has been reported in the literature. The most common pattern seen was endophytic type of growth (Figure 1). CT Scan of the patient confirmed the clinical findings (Figure 2). Exophytic growth has been observed by some [12,13].

Definitive diagnosis of Laryngeal Histoplasmosis is rarely on clinical examination, but a set of differential diagnosis can be considered [16]. The most common differential to be considered is Laryngeal Malignancy, clinically it may be impossible to ascertain the diagnosis with surety and histopathological diagnosis is always required but still lack of significant amount of symptoms, local and distant spread and less virulent nature of disease helps in formulating a hypothesis. Other differentials to be considered are Tuberculosis, Amyloidosis, Lymphoma, Syphilis and other granulomatous diseases like Sarcoidosis, Wegners etc. Though clinically these may create confusion but characteristic microscopic appearance more or less confirms the diagnosis. Rajah [17] treated, what he thought was tuberculosis in one of his patients for 10 months before the correct diagnosis, while

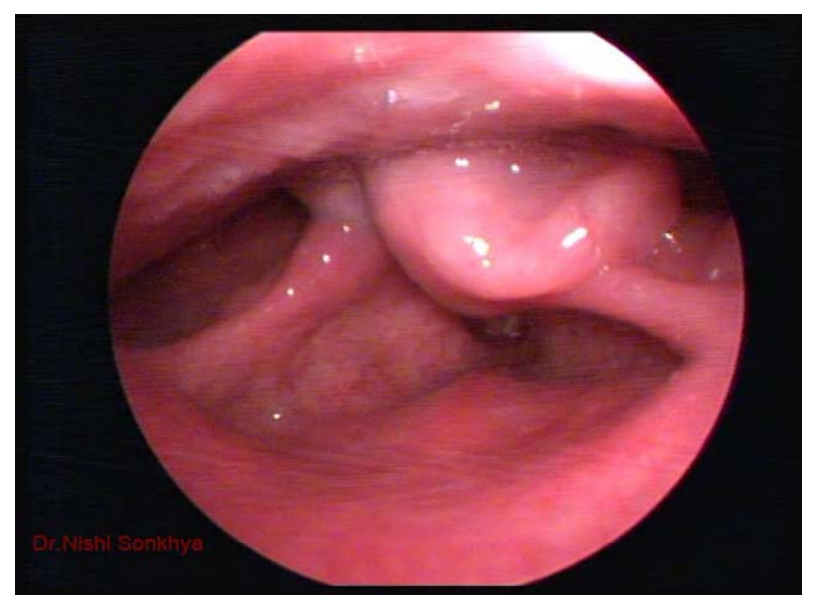

Figure 1. Telescopic view of larynx showing endophytic lesion involving left arytenoid and aryepiglottic fold.

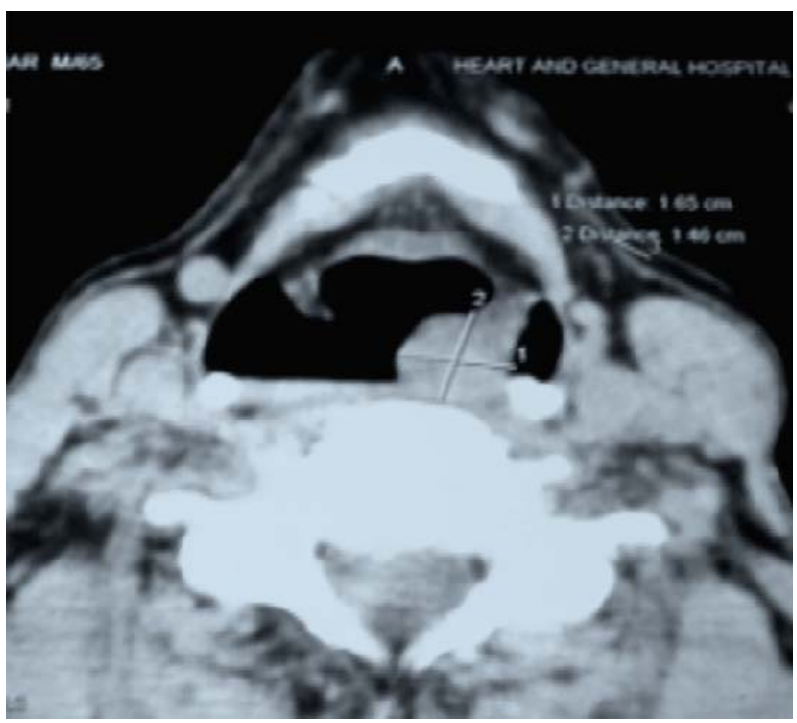

Figure 2. Contrast enhanced CT Scan Showing the enhancing mass lesion.

Staloff et al. [18] had their patient undergo two surgical procedures by another team with the diagnosis of vocal fold polyps, and later they suspected of papillomatosis. Gerber et al. [19] carried out chemotherapy and radiotherapy to treat cancer for 2 to 3 months, without improvement and achieved good results after instating proper treatment.

Diagnosis depends on clinical features and high index of suspicion especially in endemic areas. Histopathological examination with Hematoxyline \& Eosin (Figure 3), Periodic Acid Schiff (PAS) and silver methenamine stains (MSN) (Figure 4), show macrophages with yeast, granuloma with caseation and pseudoepiteliomatous hyperplasia. Culture on Sabouraud Dextrose Agar (SDA) culture medium and Immunological tests like Histoplasmin skin test and Complement fixation tests are most valuable indirect diagnostic procedure. A titer of $1: 16$ or greater are highly suggestive of infection.

Treatment is essentially medical with Itraconazole being the drug of choice in our series. Itraconazole was used in a dose of $200 \mathrm{mg}$ twice daily for 3 to 4 months. Amphotericin B was used in initial 3 cases with recurrence in 1 case after 6 months and signs of nephrotoxicity in another, but with use of Itraconazole in rest 7 cases, no recurrence or side effect was noted. Amphotericin B remains the gold standard for treatment and is highly effective, even in immunocompromised individuals; Itraconazole shows promise as an alternative to Amphotericin b for treatment of less severely ill patients [1618].

Prognosis of these patients is very good and we had full recovery in all the patients. The treatment being essentially medical both the morbidity and patient compliance is excellent. 


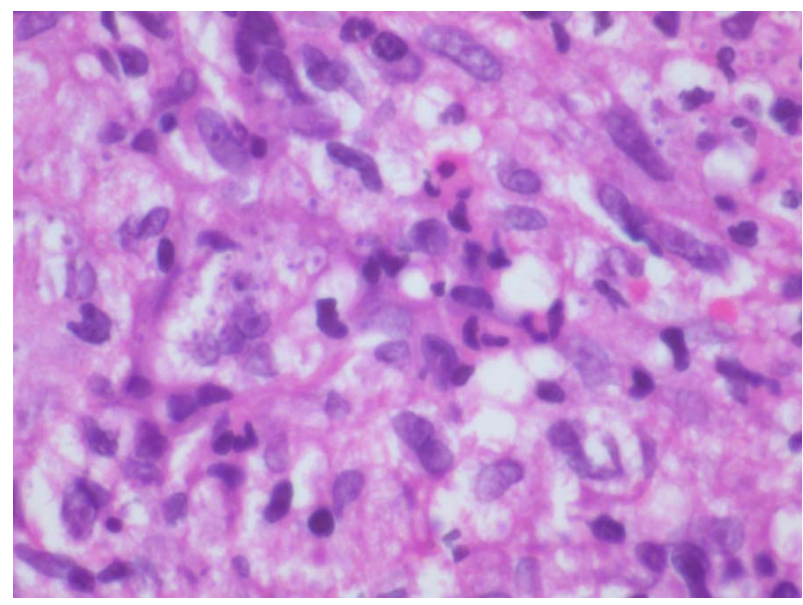

Figure 3. HE Stain $X 60 \times-h i s t i o c y t e s$ showing numerous histoplasma organism.

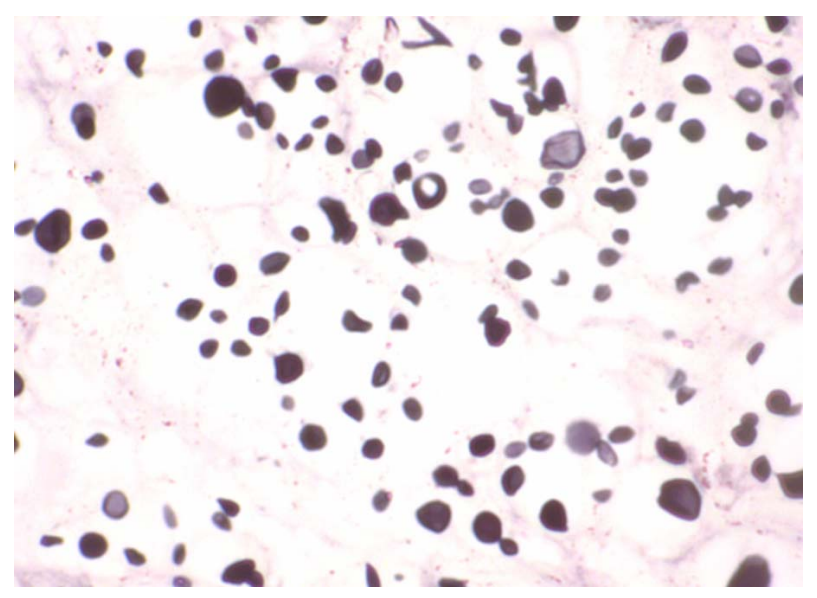

Figure 4. MSN Stain X 60×-histoplasma organism seen in the histiocytes.

\section{Conclusion}

Isolated laryngeal Histoplasmosis is a rare entity. Because of its simulation with malignancy it needs to be included in the differential diagnosis of neoplasm both benign and malignant. Our study clearly limits this kind of disease to adult males most commonly involving false cord in an Endophytic pattern. Though it looks like cancer, biopsy confirms the diagnosis. The treatment is mainly medical with Itraconazole being the drug of choice.

\section{Summary}

- Primary Laryngeal Histoplasmosis is a rare entity;

- It generally presents clinically as Benign or Malignant Laryngeal Pathology;

- Histopathological examination confirms the diagnosis;

- Treatment is essentially medical with Itraconazole showing wondrous results.

\section{REFERENCES}

[1] R. Rapini, J. Bolognia and J. Jorizzo, “Dermatology: 2-Volume Set,” St. Louis, Mosby, 2007.

[2] E. R. Bregani, T. Van Tein, T. Ceraldi, C. Delfitto and G. Fegini, "Histoplasmosis: Not Only a Tropical Disease," Recent Progress in Medicine, Vol. 91, No. 7-8, 2000, pp. 396-401.

[3] P. Tong, W. C. Tan and M. Pang, "Sporadic Disseminated Histoplasmosis Simulating Miliary Tuberculosis," British Medical Journal (Clinical Research Ed.), Vol. 287, No. 6395, 1983, pp. 822-823. doi:10.1136/bmj.287.6395.822

[4] C. A. Kauffman, "Histoplasmosis: A Clinical and Laboratory Update,” Clinical Microbiology Reviews, Vol. 20, No. 1, 2007, pp. 115-132. doi:10.1128/CMR.00027-06

[5] S. E. Roberts and F. S. Forman, "Histoplasmosis a Deficiency Disease," The Annals of Otology, Rhinology, and Laryngology, Vol. 59, No. 3, 1950, pp. 809-822.

[6] V. Kumar and A. N. Husain, “The Lung,” In; V. Kumar, A. K. Abbas and N. Fausto, Eds., Robbins and Cotran Pathological Basis of Disease, 7th Edition, WB Saunders Company, Philadelphia, 2004, pp. 712-772.

[7] M. L. Furcolow, “The Clinical Diagnosis of Histoplasmosis,” Postgraduate Medicine, Vol. 20, 1956, pp. 349364.

[8] T. C. Ferrari, J. M. Soares, J. M. Salles, J. S. Handam, R. C. Azevedo, M. A. Xavier and L. C. Faria, "Laryngeal Histoplasmosis in an Immunocompetent Patient from a Non-Endemic Region: Case Report,” Mycoses, Vol. 52, No. 6, 2009, pp. 539-540. doi:10.1111/j.1439-0507.2008.01650.x

[9] J. F. Reibel, R. A. Jahrsdoerfer, M. M. Johns and R. W. Cantrell, "Histoplasmosis of the Larynx," Otolaryngology—Head \& Neck Surgery, Vol. 90, No. 6, 1982, pp. 740-743.

[10] S. Subramanian, A. H. Abdullah and I. Hairuzah, "Histoplasmosis of Larynx," The Medical Journal of Malaysia, Vol. 60, No. 3, 2005, pp. 386-388.

[11] F. P. Sobrinho, M. D. Negra, W. Queiroz, U. J. Ribeiro, S. Bittencourt and G. B. Klautau, "Histoplasmosis of the Larynx,” Brazilian Journal of Otorhinolaryngology, Vol. 73, No. 6, 2007, pp. 857-861.

[12] P. Katoch and S. Bhardwaj, "Primary Laryngeal Histoplasmosis: Case Report,” JK Science, Vol. 11, No. 2, 2009, pp. 89-90.

[13] J. O. Donegan and M. D. Wood, "Histoplasmosis of Larynx,” Laryngoscope, Vol. 94, No. 2, 1984, pp. 206-209. doi:10.1288/00005537-198402000-00011

[14] A. M. Phatak, I. Bhattacharya, V. Misra, A. M. Prabhu and U. Natraj, "Disseminated Histoplasmosis Mimicking Laryngeal Carcinoma from Central India: A Case Report," Indian Journal of Pathology and Microbiology, Vol. 49. No. 3, 2006, pp. 452-454.

[15] L. J. Wheat, "Histoplasmosis in Indianapolis,” Clinical 
Infectious Diseases, Vol. 14, Suppl. 1, 1992, pp. 91-99. doi:10.1093/clinids/14.Supplement 1.S91

[16] J. Wheat, "Histoplasmosis: Recognition and Treatment," Clinical Infectious Diseases, Vol. 19, Suppl. 1, 1994, pp. 19-27. doi:10.1093/clinids/19.Supplement_1.S19

[17] V. Rajah, "Histoplasmosis of the Oral Cavity, Oropharynx and Larynx," The Journal of Laryngology and Otology Vol. 107, No. 1, 1993, pp. 58-61.

doi:10.1017/S0022215100122169
[18] R. T. Staloff, A. Wilborn, A. Prestipino, M. Hawkshaw, J. H. Reinhardt and J. Cohn, "Histoplasmosis of the Larynx," American Journal of Otolaryngology, Vol. 14, No. 3, 1993, pp. 199-205. doi:10.1016/0196-0709(93)90030-B

[19] M. E. Gerber, J. D. Rosdeutscher, A. M. Seiden and T. A. Tami, "Histoplasmosis: The Otorynolaringologist's Perspective," Laryngoscope, Vol. 105, No. 9, 1995, pp. 919923. doi:10.1288/00005537-199509000-00009 